\title{
Osteoinductive Properties of Secretome of Human Mesenchymal Stem Cells, Obtained with Automatic Cell Culture System
}

\author{
S. A. Aleksandrova ${ }^{a, *}$, Yu. A. Nashchekina ${ }^{a}$, S. V. Nadezhdin ${ }^{b}$, S. A. Vasiliev ${ }^{c}$, R. R. Savchenko ${ }^{c}$, \\ L. A. Pokrovskaya ${ }^{d}$, M. I. Blinova ${ }^{a}$, N. A. Mikhailova ${ }^{a}$, and M. G. Khotin ${ }^{a}$ \\ ${ }^{a}$ Institute of Cytology, Russian Academy of Sciences, St. Petersburg, 194064 Russia \\ ${ }^{b}$ Belgorod National Research University, Belgorod, 308015 Russia \\ ${ }^{c}$ Scientific Research Institute of Medical Genetics, Tomsk National Research Medical Center, Russian Academy of Sciences, \\ Tomsk, 634050 Russia \\ ${ }^{d}$ National Research Tomsk State University, Tomsk, 634050 Russia \\ *e-mail: alekssvet2205@gmail.com
}

Received December 2, 2019; revised January 26, 2020; accepted January 28, 2020

\begin{abstract}
The secretome of human mesenchymal stem cells (MSCs) after osteogenic differentiation of FetMSC cells in vitro was obtained to develop tissue engineering technologies for bone tissue. For standard secretome samples a large number of FetMSC cells $\left(7 \times 10^{8}\right.$ cells $)$ were cultured in serum-free conditioned medium (SFCM). All steps of cell processing were performed in the CompacT SelecT automatic system (Sartorius, United Kingdom). The SFCM was concentrated by ultrafiltration, then subjected to dialysis and dried in a vacuum rotary evaporator. The osteoinductive properties of SFCM concentrate (SFCMC) were tested on FetMSC cells by means of adding it to the growth medium at different concentrations. There were no changes in cell morphology during cultivation in the presence of SFCMC. Analysis of the transcription factors Runx 2 and YAP1 (markers of osteogenic differentiation) expression using immunofluorescence staining and real-time polymerase chain reaction (RT-PCR) showed an increase in their level of expression in the presence of SFCMC. These findings demonstrate that it is possible to use SFCMC obtained from osteogenic MSCs to induce differentiation of other (undifferentiated) MSCs. The results are useful for the development of cell therapy products for bone restoration based on MSCs secretome.
\end{abstract}

Keywords: mesenchymal stem cells of human bone marrow, cell line FetMSC, osteogenic differentiation, Runx2, YAP1, secretome, automatic cell culture system

DOI: $10.1134 / \mathrm{S} 1990519 X 20060024$

\section{INTRODUCTION}

Currently, tissue engineering of bone tissue is directed at creating three-dimensional structures, socalled "grafts," which are a scaffold populated by stem cells. Various materials are employed to produce scaffolds, often modified with extracellular matrix proteins, growth factors, or genetic constructs to impart osteoinductive properties to them (Deev et al., 2015). Improved delivery and preservation of growth factors is provided by their encapsulation in drug delivery systems (microspheres, liposomes, hydrogels, etc.) and their introduction into scaffolds (Porter et al., 2009). Such incorporation of bioactive substances solves several basic problems: targeted delivery of growth factors with optimal concentration inside the implant, preser-

Abbreviations: SFCM—serum-free conditioned medium; DMdifferentiation medium; SFCMC-SFCM-concentrate; BMbone marrow; MSCs-mesenchymal stem cells; RT-PCRreal-time polymerase chain reaction; FCS-fetal calf serum; PBS - phosphate buffered saline. vation of the biological activity of the molecules, and controlled release of substances for the required period of time. However, the optimal composition of the content is still under discussion (Sundelacruz and Kaplan, 2009). Many processes occur during regeneration, and so the use of one or more factors is not enough for successful tissue repair.

In the past few decades, mesenchymal stem cells (MSCs) isolated from human tissues and cultivated in vitro are under active investigation aimed at harnessing them for regenerative medicine (Murphy et al., 2013; Mizukami and Swiech, 2018). The main MSCs properties are high proliferative activity and the capacity for directed differentiation into connective tissue cells, as well as production of various substances involved in the regulation of cellular functions associated with tissue renewal (Da Silva Meirelles et al., 2009; Caplan, 2019). In recent years, with the help of new transcriptomic and proteomic approaches it has been revealed that MSCs synthesize a complex of biologically active substances (secretome) that coordinate 
many biological functions of the organism, such as growth, division, differentiation, and cell death (Baglio et al., 2012; Ivanova et al., 2016). The specific composition of MSCs secretome varies depending on the donor and tissue origin and alters with certain factors exposed to cells (Assoni et al., 2017).

Many studies have shown that the cellular microenvironment has a great influence on the secretome composition (Kusuma et al., 2017). Analysis of MSCs secretome revealed a number of proteins, the expression of which changed during osteogenic differentiation. Kim et al. (2013) identified 315 proteins, of which more than 200 were differentially expressed during osteogenesis. It has been shown (Kulterer et al., 2007) that, in the first 4-5 days, transcription factors and genes associated with proliferation were predominantly expressed; then (up to 14 days), the genes responsible for the production of the extracellular matrix were; and, from 14 days on, the genes responsible for the mineralization of the matrix.

In a number of studies on the therapeutic application of MSCs, it was found that most of the clinical effects observed during transplantation MSCs were achieved mainly due to the produced paracrine factors. Thus, it was demonstrated that the use of factors produced by cells in the process of differentiation (or an entire conditioned medium) is a signal for the differentiation of stem cells of damaged tissues (Zhong et al., 2019). In addition, it is also known that these factors affect on proliferation, chemotaxis, and angiogenesis, which accelerate reparative processes (Deev et al., 2015). Therefore, the idea was put forward that MSCs can be replaced with a secretome without significant loss of clinical effect. Another argument for replacing cells with their secretome is represented by the intractable questions that exist about the standardization of cellular material, as well as the risks of tumor transformation (Stanovici et al., 2016).

To develop a medical technology using a complete MSCs secretome (or its particular fractions) for the control of regeneration, the problem of obtaining a sufficiently large amount of MSCs secretome and its standardization arises. When using this technology to repair damaged bone tissue, the secretome must have osteoinductive properties.

The aim of this work was to obtain a lyophilized concentrate of serum-free conditioned medium (SFCM) after human MSCs (FetMSC cell line) differentiation in the osteogenic direction and examine its osteoinductive properties in terms of the effect on cell morphology and their osteogenic differentiation.

\section{MATERIALS AND METHODS}

\section{Cultivation and Induction of Osteogenic Differentiation of Human Bone Marrow MSCs Using the Automatic Station CompacT SelecT}

We used the FetMSC cell line (Collection of Vertebrate Cell Cultures, Institute of Cytology of the Russian Academy of Sciences, St. Petersburg), obtained from MSCs of human embryonic bone marrow (Krylova et al., 2012). Cells were thawed and cultured on $75-\mathrm{cm}^{2}$ flasks (T75) under the good manufacturing conditions of the Laboratory of Biomedical Technologies and Testing with a pilot production facility (Center of Cell Technologies of the Institute of Cytology). Cells were multiplied in an automatic CompacT SelecT cell culture station (Sartorius, United Kingdom) (Fig. 1) for standardize all stages of cultivation and obtain a serum-free conditioned medium (SFCM). All subsequent cellular processing was performed using the capabilities of this robotic system. For this purpose, we have developed algorithms for sequential automated manipulations. The sequential steps of cell processing and media exchange using CompacT SelecT are presented in Table 1.

The cells were cultured in DMEM medium under aseptic controlled conditions: a temperature of $37^{\circ} \mathrm{C}$, humidity of $98 \%$, and composition of the gas medium of $5 \% \mathrm{CO}_{2}$. For cell growth, a DMEM/F12 medium (Gibco, USA) containing 10\% bovine embryo serum (SEC) (Gibco, USA) was used. The CompacT SelecT system determines the confluence of cells in the culture at a frequency set by the operator, and, when the set value is reached, it performs an automated cell subculture. The collection of conditioned medium, cell counting, and assessment of their viability were also done in an automatic mode. This approach ensured homogeneity of the obtained cell population and the standardization of the conditioned medium collected from a large volume of cells into a sterile vessel for subsequent analysis.

After accumulating a sufficient number of cells the culture medium was replaced with differentiation medium (DM), containing a complete DMEM/F12 culture medium supplemented with dexamethasone $(0.1 \mu \mathrm{M})$, ascorbic acid $(50 \mu \mathrm{M})$, and sodium $\beta$-glycerophosphate $(10 \mathrm{mM})$ for stimulation differentiation of MSCs in the osteogenic direction (Pittenger et al., 2001). After $72 \mathrm{~h}$, the culture medium was removed, the cell monolayer was washed three times with PBS, supplemented with serum-free DMEM/F12 growth medium, and cultured for $72 \mathrm{~h}$. The medium was then automatically collected under aseptic conditions in a sterile container. This medium was assigned as SFCM, and its components secreted by differentiated MSCs were designated as secretome of osteogenic MSCs. During the cultivation, the induced cells secreted into the growth medium metabolites produced in the process of osteogenic differentiation. After collection of SFCM, the cell viability and level of 


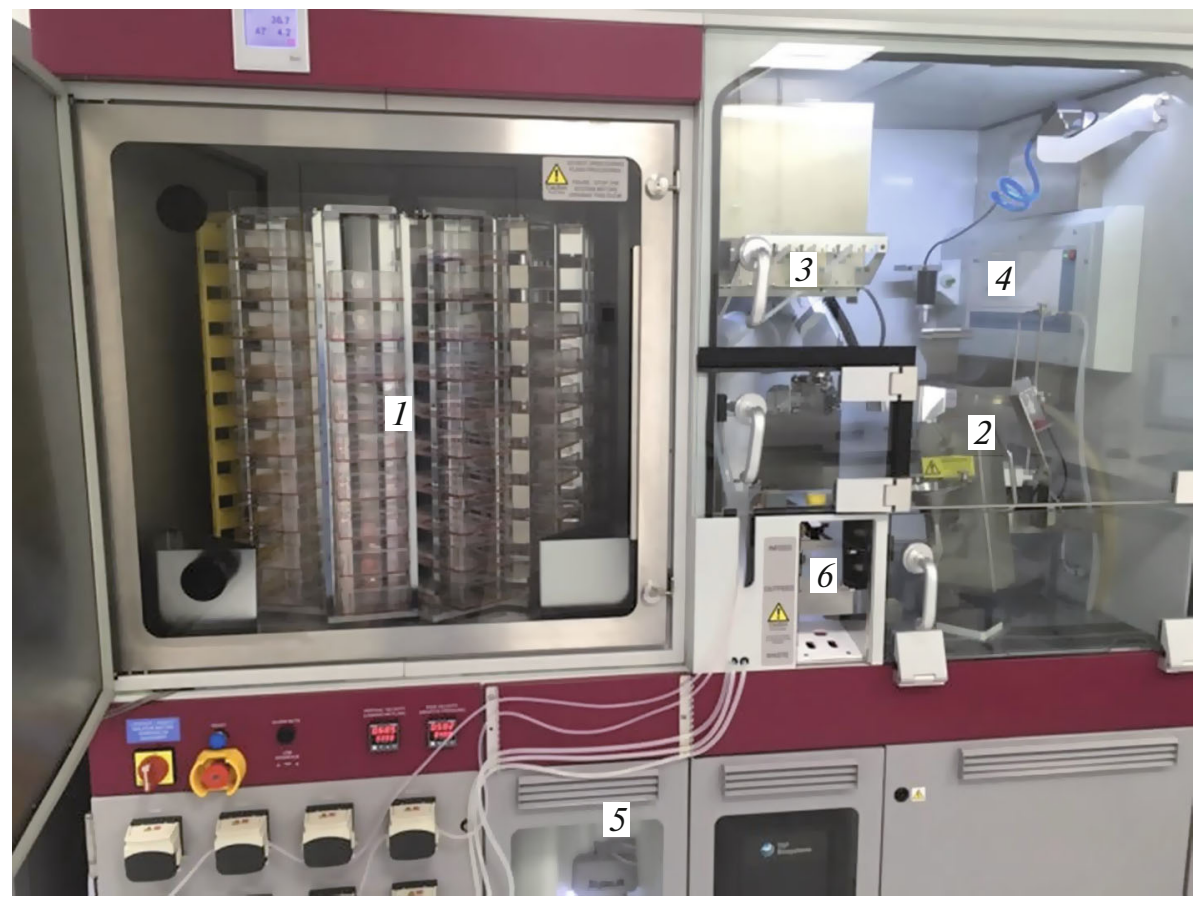

Fig. 1. CompacT SelecT automatic system for cell cultivation and analysis (Sartorius, United Kingdom). Working areas of the system: (1) incubator with loaded flasks, (2) robotic arm of the device for handling solutions and flasks with cells, (3) area for bottling nutrient media and other liquids, (4) IncuCyte module (Essen Biosciences, United States), (5) ViCell cell counter (Beckman, United States), and (6) gate for flask transfer to and from the system.

expression of the transcription factors Runx2 and YAP1 activated at the early stages of osteogenic induction (Yagi et al., 1999; Komori et al., 2011), were assessed with real-time polymerase chain reaction (RT-PCR) (see the method below).

\section{Preparation of the Serum-Free Conditioned Medium Concentrate from Induced MSCs (SFCMC)}

The collected SFCM was centrifuged at $1660 \mathrm{~g}$ for $10 \mathrm{~min}$. The obtained supernatant was passed through a filter with $0.22-\mu \mathrm{m}$ pores and then concentrated by ultrafiltration in tangential flow in the Vivaflow ${ }^{\circledR} 200$ system (Sartorius, Germany) using a membrane module with a cutoff threshold for molecules with a molecular weight of $10 \mathrm{kDa}$. The resulting solution was dialyzed using the same system by adding deionized water to the sample container (diafiltration). The concentrate purified from salts was dried in a Rotavapor ${ }^{\circledR}$ R-210 vacuum rotary evaporator (Buchi, Germany) and placed in a microcentrifuge tube. Experiments on SFCMC production were performed in the Research Laboratory "Cellular, Assisted Reproductive, and DNA Technologies" (Belgorod State National Research University).

The lyophilized concentrate (SFCMC) was diluted in serum-free DMEM/F12 medium (Rosmedbio, Russia) and sterilized by passing through a filter with $22-\mu \mathrm{m}$ pores under aseptic conditions. This stock solution, with a concentration of $33.33 \mathrm{mg} / \mathrm{mL}$, was further added to the nutrient medium (in the absence of fetal calf serum (FCS)).

\section{Intravital Study of MSCs Morphology}

FetMSC cells (passages 4-5 after decryopreservation) were seeded into 24-well plates with 100 thousand cells per well and cultivated for 1 and 7 days under standard conditions. To examine the possible effect of SFCMC on the cell morphology, the stock solution was added to the culture medium (not containing FCS) in concentrations of 0.33 and $0.66 \mathrm{mg} / \mathrm{mL}$. Digital imaging of cell cultures in real time was carried out with an Eclipse TS100 inverted light microscope (Nikon, Japan).

\section{Immunofluorescent Detection of Transcription Factors}

FetMSC cells were seeded on glasses, placed on the bottom of the wells of 24 -well plates $\left(10^{5}\right.$ cells per glass), and cultured for 7 days under standard conditions. To study the osteoinductive properties of SFCMC, it was added to the culture medium without FCS at concentrations of 0.33 or $0.66 \mathrm{mg} / \mathrm{mL}$. Cells growing in DM were used as a positive control; cells maintained under standard conditions served as a negative control. On the seventh day of cultivation, the growth medium was discarded; the cells were washed 
Table 1. Cell processing steps using the automatic station CompacT SelecT

\begin{tabular}{|c|c|c|c|c|}
\hline Step & Description & Culture medium & CompacT SelecT manipulations & Control parameters \\
\hline $\begin{array}{l}\text { 1. Growth of } \\
\text { FetMSC cells }\end{array}$ & $\begin{array}{l}\text { Mass-cultivation of } \\
\text { cells in order to obtain } \\
50 \text { T175 flasks at pas- } \\
\text { sage } 5\end{array}$ & $\begin{array}{l}\text { CM: } \\
\text { DMEM/F12 + 10\% } \\
\text { FBS }\end{array}$ & $\begin{array}{l}\text { CF control is performed every } \\
12 \text { hours in each vial using the } \\
\text { IncuCyte module. When the CF } \\
\text { reaches } 85 \% \text {, the cells are subcul- } \\
\text { tured } 1: 5 \text {. Cell vitality and num- } \\
\text { ber are controlled by ViCell cell } \\
\text { counter. Cells passaging are } \\
\text { included: draining the medium } \\
\text { from the flasks, washing with PBS, } \\
\text { adding trypsin with shaking the } \\
\text { flask, adding CM, mixing the cell } \\
\text { suspension by pipetting and trans- } \\
\text { ferring to new flasks }\end{array}$ & $\begin{array}{l}\text { Cell vitality: } \\
95-100 \% \text {. } \\
\text { The number of cells } \\
\text { per } 1 \text { bottle of T175: } \\
(12-16) \times 10^{6} \text {. } \\
\text { Plotting a growth } \\
\text { curve for each bottle }\end{array}$ \\
\hline $\begin{array}{l}\text { 2. Replacement } \\
\mathrm{CM} \text { to DM }\end{array}$ & $\begin{array}{l}\text { Simultaneous substitu- } \\
\text { tion CM in } 50 \mathrm{~T} 175 \\
\text { flasks with cells }(\mathrm{CF}= \\
40-60 \%) \text { to } \mathrm{DM}\end{array}$ & $\begin{array}{l}\text { DM: } \\
\text { DMEM/F12 + 10\% } \\
\text { FBS + 0.1 } \mu \mathrm{M} \text { dexa- } \\
\text { methasone, }+50 \mu \mathrm{M} \\
\text { ascorbic acid, } \\
+10 \text { mM sodium } \beta- \\
\text { glycerophosphate }\end{array}$ & $\begin{array}{l}\text { Evaluation of CF before replace- } \\
\text { ment CM is estimated by Incu- } \\
\text { Cyte module. Adding DM and } \\
\text { placing the vials in an incubator } \\
\text { for } 72 \mathrm{~h} \text {. Assessment of cell mor- } \\
\text { phology during differentiation }\end{array}$ & $\begin{array}{l}\text { CF before } \\
\text { replacement CM to } \\
\text { DM: } 40-65 \%\end{array}$ \\
\hline $\begin{array}{l}\text { 3. Replacement } \\
\text { DM to SFM }\end{array}$ & $\begin{array}{l}\text { Simultaneous substitu- } \\
\text { tion DM to SFM in } \\
50 \mathrm{~T} 175 \text { flasks with cells } \\
\text { in } 72 \mathrm{~h} \text { of induction }\end{array}$ & $\begin{array}{l}\text { SFM: } \\
\text { DMEM/F12 }\end{array}$ & $\begin{array}{l}\text { CF assessment before changing } \\
\text { the medium (IncuCyte module). } \\
\text { Washing the vials with PBS cells } \\
3 \text { times, adding SFM and placing } \\
\text { the vials in an incubator for } 72 \mathrm{~h}\end{array}$ & $\begin{array}{l}\text { CF before } \\
\text { by changing the } \\
\text { environment: } \\
80-90 \%\end{array}$ \\
\hline $\begin{array}{l}\text { 4. Collection of } \\
\text { SFM (SFCM) }\end{array}$ & $\begin{array}{l}\text { SFCM from } 50 \text { T175 } \\
\text { flasks }\end{array}$ & & $\begin{array}{l}\text { Sequential replacement of SFCM } \\
\text { from } 50 \text { flasks into a clean con- } \\
\text { tainer (by means of robotic arm). } \\
\text { Sealing the container and handing } \\
\text { it over to the operator. Selectively } \\
\text { medium is collected from } 5 \text { vials } \\
\text { and cells are removed by trypsin } \\
\text { treatment. Their vitality is ana- } \\
\text { lyzed by the ViCell counter }\end{array}$ & $\begin{array}{l}\text { Cell vitality: } \\
90-100 \%\end{array}$ \\
\hline
\end{tabular}

CF-confluence; CM-culture medium; DM-differentiation medium, FBS-fetal calf serum; PBS-phosphate buffered saline; SFCM-serum-free conditioned medium; SFM-serum-free medium.

with PBS and fixed in a $10 \%$ formalin solution (BioVitrum, Russia). Mouse monoclonal antibodies to Runx2 protein (ab76956) and rabbit monoclonal antibodies to YAP1 (ab205270; Abcam, United Kingdom) were used as primary antibodies. Fluorescently labeled (AlexaFluor® 488) goat polyclonal antibodies to mouse IgG (ab150113) and goat polyclonal antibodies (AlexaFluor® 594) to rabbit IgG (ab150080) (Abcam, United Kingdom) were used, respectively, as secondary antibodies. After incubation with secondary antibodies, the cells were mounted in DAPI-FluoroShield medium (Vector Laboratories, United States) containing DAPI (the intercalating dye for staining cell nuclei).

CELL AND TISSUE BIOLOGY Vol. $14 \quad$ No. 62020
Cells imaging was performed with a $\mathrm{ZOE}^{\mathrm{TM}}$ inverted confocal microscope Fluorescent Cell Imager (United States).

\section{Real-Time Polymerase Chain Reaction}

To detect the expression of the runx 2 and yap 1 genes, FetMSC cells were seeded into 24-well plates, $10^{5}$ cells per well, and cultivated for 3,7 , and 14 days under standard conditions. Osteoinductive properties of SFCMC were tested by its addition in serum-free culture medium at concentrations of 0.33 or $0.66 \mathrm{mg} / \mathrm{mL}$. Cells cultivated in DM and Hos human osteosarcoma cells (TE85, clone F5) from the Collection of Vertebrate Cell Cultures, Institute of Cytology, 
Russian Academy of Sciences (St. Petersburg) were used as positive controls. Cells cultivated under standard conditions served as negative control.

RNA from cells was isolated using Lira reagent (Biolabmix, Russia) containing phenol and guanidine thiocyanate. Reverse transcription was performed with the RTM-MuLV-RH Reverse Transcription Kit (Biolabmix, Russia). Oligonucleotide primers to runx2 gene (5'-ACCGAGACCAACAGAGTCATTTA-3', 3'-GTGTCACTGTGCTGAAGAGG$\left.5^{\prime}, 119 \mathrm{bp}\right)$ and to yap 1 gene (5'-AACAGCAAGAACTGCTTCGG-3', 3'-GCAGGGCTAACTCCTGACAT-5', $84 \mathrm{bp}$ ) were selected by the authors of this work. Primers to ppia gene sequences (5'-AGACAAGGTCCCAAAGAC-3', 3'-ACCACCCTGACACATAAA-5', 118 bp) and hprt 1 gene (5'GACCAGTCAACAGGGGACAT-3'， 3'-CCTGACCAAGGAAAGCAAAG-5', 132 bp) were used as reference. Highly purified primers were synthesized at OOO Biosset (Novosibirsk, Russia). RT-PCR was performed using the Maxima SYBRGreen kit (ThermoScientific, United States) in AriaMx amplifier (Agilent Technologies, United States).

Gene expression analysis was performed at the Core Medical Genomics Facility of the Tomsk National Research Medical Center (Tomsk NRMC) of the Russian Academy of Sciences.

\section{RESULTS AND DISCUSSION}

\section{Production of SFCMC from Human Osteogenic MSCs}

For standard cultivation of FetMSC, a special algorithm was developed that constructed the program for the robot. The whole process of scaling and collecting of SFCM described below was carried out with this program. Cells were cultivated in the CompacT SelecT system for five passages from one T75 flask transferred to the robot. The system performed cell subculture upon reaching the confluence of $85 \%$. For this, the cell confluence in culture was monitored every $12 \mathrm{~h}$. The cells were subcultured in a $1: 5$ ratio, and the medium was replaced at least once within 3 days. By the 5 th passage, $7 \times 10^{8}$ cells were accumulated on $50 \mathrm{~T} 175$ flasks. The viability of cells ranging in size from 10 to $50 \mu \mathrm{m}$ was $98-100 \%$ during the entire processing. After obtaining 50 flasks of cells with a confluence of $50-60 \%$, the system replaced the growth medium with the induction medium (DM) for MSCs differentiation in the osteogenic direction. After cell differentiation $(72 \mathrm{~h})$, the medium was exchanged with the serum-free medium and the cells were cultured for $72 \mathrm{~h}$. A total of $1000 \mathrm{~mL}$ of SFCM was collected.

The cell shape during the entire time of the experiment remained fibroblast-like, and the cells formed a monolayer (Fig. 2). After collecting SFCM, two randomly selected flasks were treated with lysis buffer for RNA isolation immediately after the medium collec- tion. Next, we studied the expression of the runx 2 and yap 1 genes in cells in comparison with positive controls (cells in DM and Hos cells). Expression of the runx 2 gene in the positive control and Hos cells was five and three times higher than in the negative control, respectively. Expression of the yap 1 gene in the positive control was twice that in the negative control. In Hos cells, it did not differ from the negative control. In FetMSC cells after SFCM collection, the level of expression of the runx2 gene was twice as high as in the negative control, but did not reach the level of expression in the positive control. Expression of yap 1 was 1.7 times higher than in the negative control, practically reaching the corresponding value in the positive control.

\section{Osteoinductive Properties of SFCMC \\ Influence of SFCMC on MSCs Morphology}

Live cell imaging in the presence of SFCMC did not reveal obvious morphological differences between the samples (not shown). The cells actively proliferated and after 7 days of cultivation, created a dense monolayer. Supposedly, the rather clear morphological changes that occur during osteogenesis have not yet appeared during this time.

\section{Accumulation of Transcription Factors Runx2 and YAP1 in the Presence of SFCMC}

The cells bound Runx2 antibodies and exhibited green fluorescence in their cytoplasm (Fig. 3). Fluorescence was observed in cells in the presence of SFCMC at both concentrations $(0.33$ and $0.66 \mathrm{mg} / \mathrm{mL}$ ) for 7 days at approximately the same level (Figs. 3c, 3d). Its level was lower than in the osteogenic medium (Fig. 3b), but higher than in the growth medium (Fig. 3a). At the same time, staining was detected unevenly on the preparations; therefore, it was difficult to make a quantitative comparison.

Cells stained with antibodies to YAP1 exhibited fluorescence in nuclei (Fig. 4). On preparations of cell cultured in the presence of SFCMC, the majority of nuclei had a red color (Figs. 4c, 4d), in contrast to the negative control (Fig. 4a), but less than cells cultured in the osteogenic medium (Fig. 4b).

\section{Detection of runx 2 and yap 1 Gene Expression by $R T-P C R$}

Cultivation of cells in the presence of SFCMC increased expression of the runx2 gene already on the third day (2.5 times at an SFCMC concentration of $0.66 \mathrm{mg} / \mathrm{mL}$ and five times with $0.33 \mathrm{mg} / \mathrm{mL} \mathrm{SFCMC}$ (Fig. 5). The maximum expression was observed after 7 days (45 and 12 times higher than the negative control at an SFCMC concentration of 0.66 and $0.33 \mathrm{mg} / \mathrm{mL}$, respectively). On the third and seventh days, the expression exceeded even the values of the 
Passaging

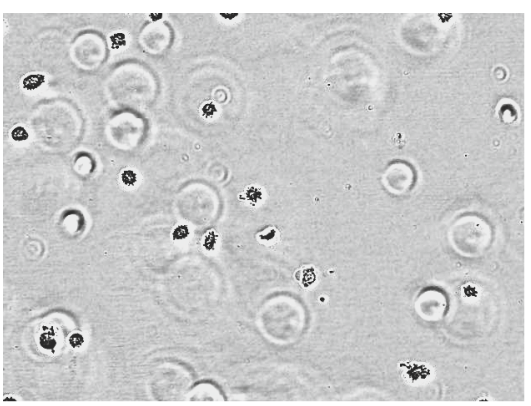

Serum-free culture medium

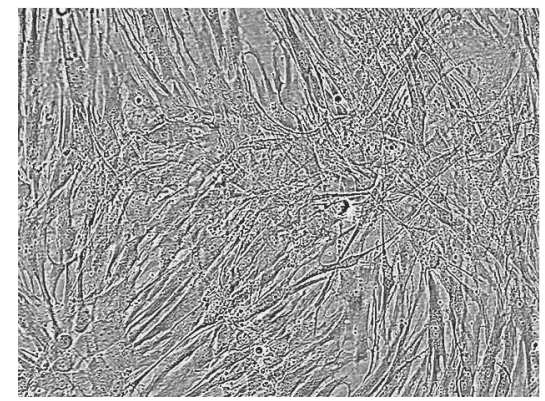

Culture medium

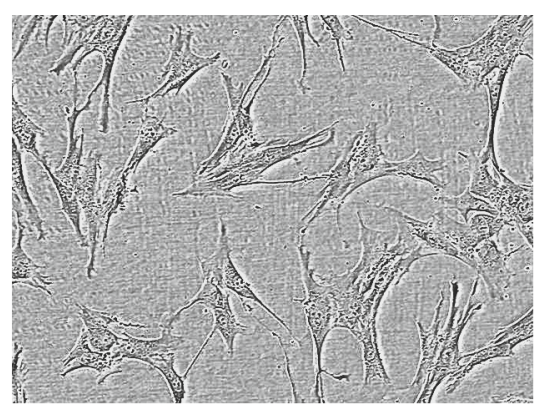

After collection of SFCM

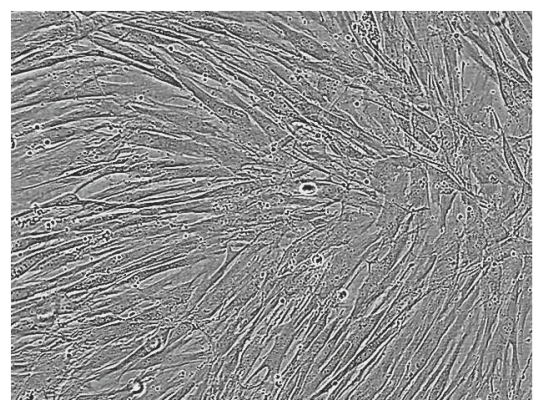

Differentiation medium

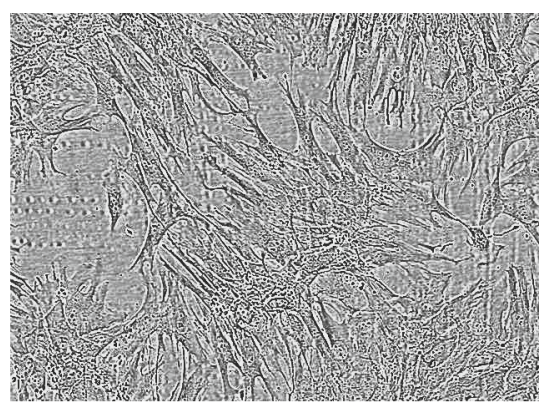

Cell growth of FetMSC

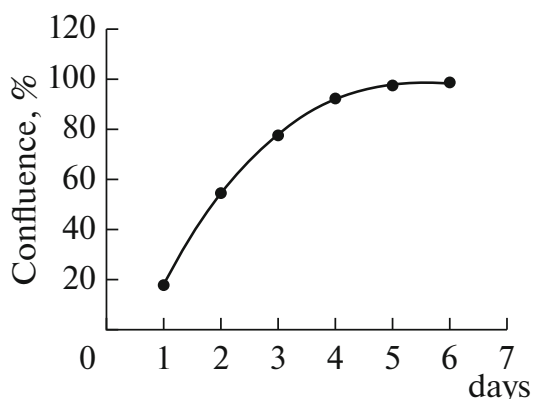

Fig. 2. FetMSC cells during cultivation in the CompacT SelecT robotic system. Cell growth curve after the last subculture. Subcultured cells have fibroblast-like morphology. Inverted microscopy. Ob.: 10×.
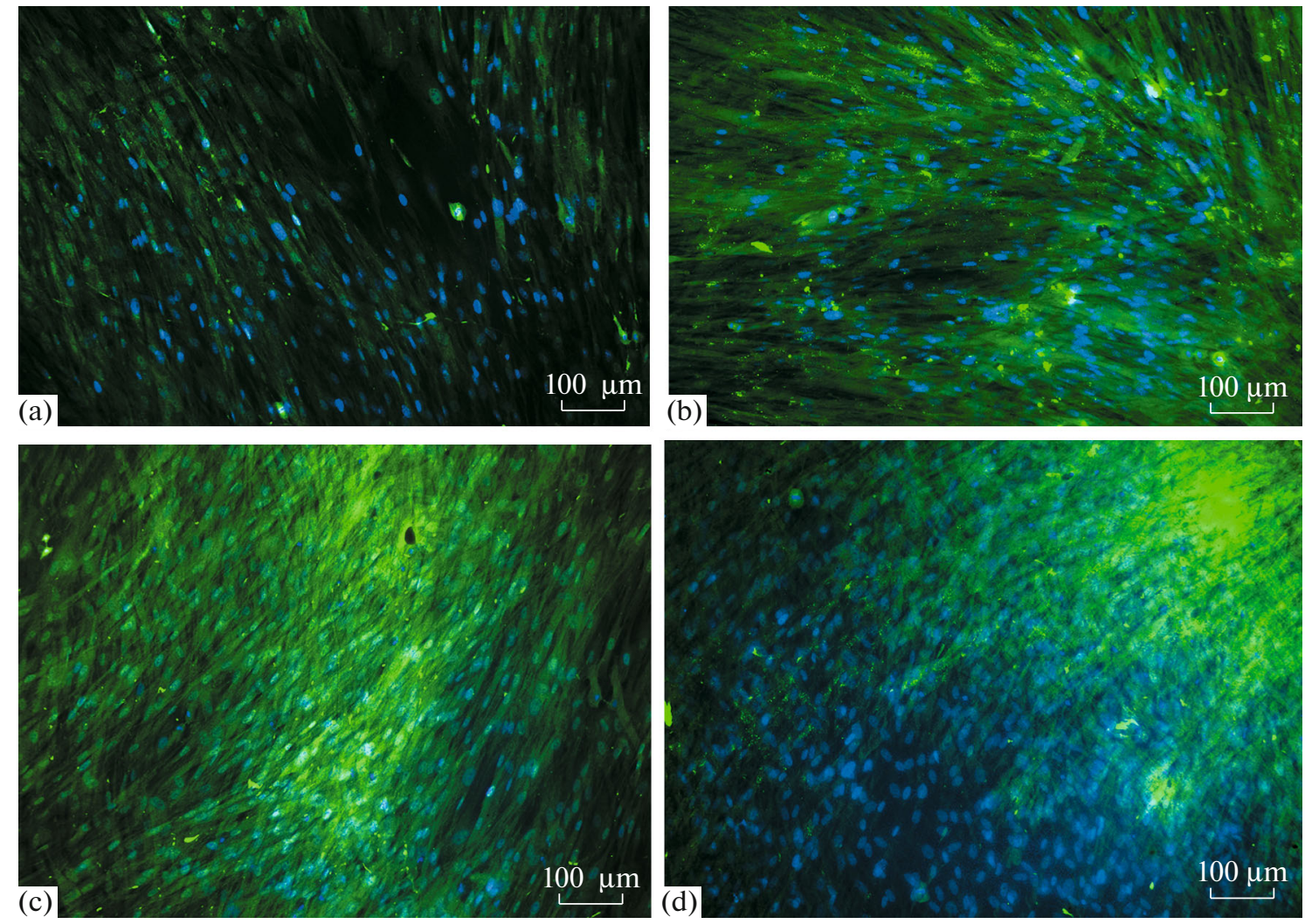

Fig. 3. Immunofluorescent staining of Runx 2 protein (green color) with monoclonal antibodies in FetMSC cells after cultivation in (a) growth and (b) osteogenic media, as well as in the presence of SFCMC at a concentration of (c) $0.33 \mathrm{and}$ (d) $0.66 \mathrm{mg} / \mathrm{mL}$ for 7 days. Cell nuclei are stained with DAPI (blue). Confocal microscopy. Ob: $20 \times$.

CELL AND TISSUE BIOLOGY Vol. $14 \quad$ No. $6 \quad 2020$ 

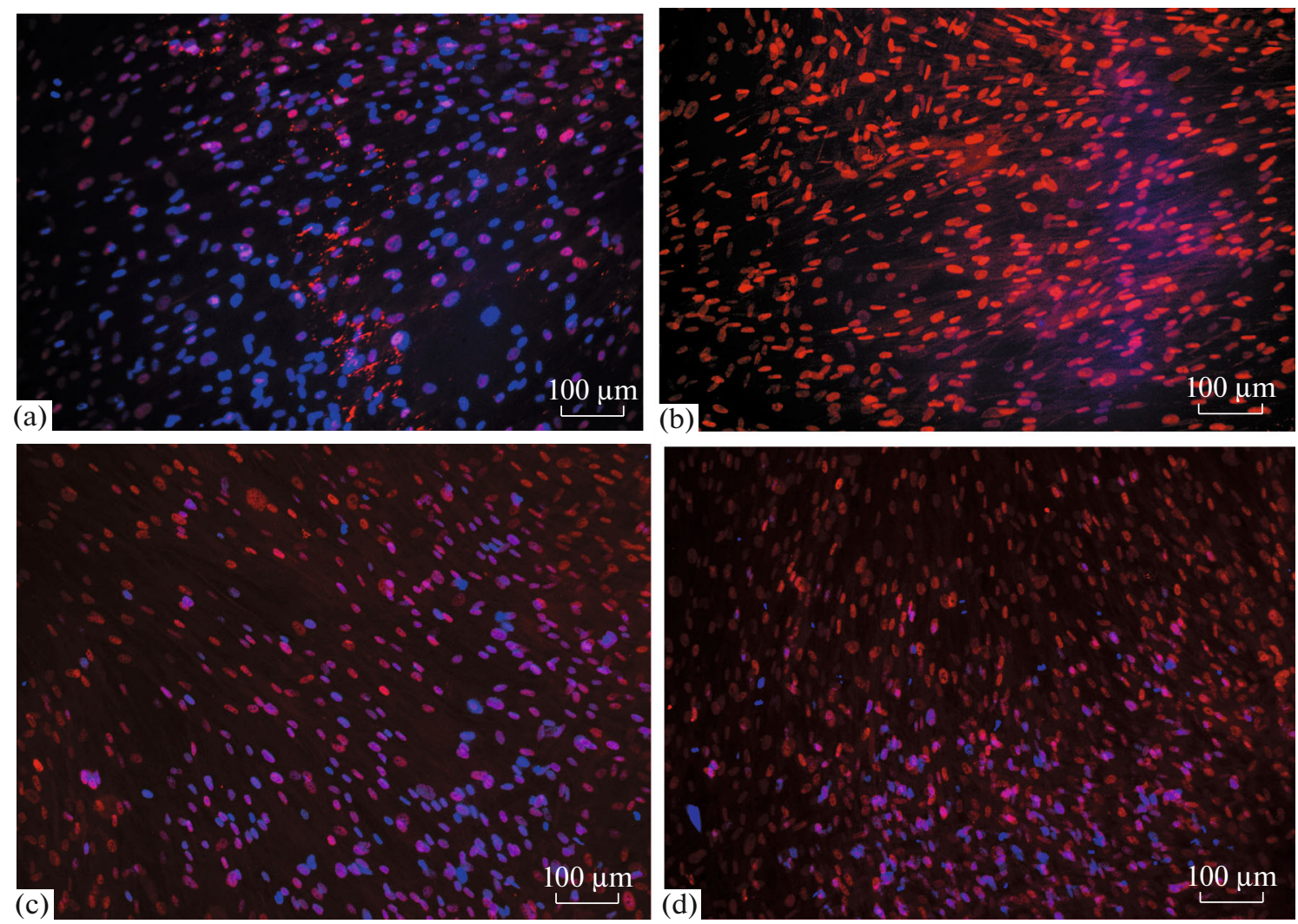

Fig. 4. Immunofluorescent staining of the YAP1 protein (red color) with monoclonal antibodies in FetMSC cells after cultivation in (a) growth and (b) osteogenic media, as well as in the presence of SFCMC at a concentration of (c) $0.33 \mathrm{and}$ (d) $0.66 \mathrm{mg} / \mathrm{mL}$ for 7 days. Cell nuclei are stained with DAPI (blue). Confocal microscopy. Ob: $20 \times$.

positive control (DM) (by two to five and two to seven times, respectively). As in the positive control, on the 14 th day of the experiment, the expression of the runx2 gene decreased; nevertheless, it remained three times higher than in the negative control. Expression of the yap 1 gene was characterized by slightly different dynamics. In cells cultivated with SFCMC, it only slightly increased relative to the negative control on the third day (1.6 times at an SFCMC concentration of $0.66 \mathrm{mg} / \mathrm{mL}$ ). On the seventh day of cultivation, on the contrary, the expression exceeded the corresponding value of the negative control only for the SFCMC concentration of $0.33 \mathrm{mg} / \mathrm{mL}$ (3.2 times relative to the negative control and 1.4 times relative to the positive control). Finally, after 14 days, the expression exceeded the values of the negative control for both concentrations of SFCMC (3.3 times at $0.66 \mathrm{mg} / \mathrm{mL}$ and 2.9 times at $0.33 \mathrm{mg} / \mathrm{mL}$ ), being slightly lower than the positive control values (1.1-1.3 times). For the other reference gene, hprt1, similar results were obtained.

Thus, the dynamics of the runx 2 gene expression indicates that SFCMC has an osteoinductive activity that exceeds that of known methods of osteoinduction. At the same time, SFCMC has a slightly lower potential for inducing yap 1 gene expression.

\section{CONCLUSIONS}

Large-scale cell culturing is usually used to obtain a standard cell mass for further research or banking. In this work, the method was applied to manufacture an experimental sample of the SFCM conditioned medium containing the secretome of osteogenic cells. The developed technology includes not only the growth of cell mass, but also further experimental cultivation of cells under particular conditions (medium condition). As a result, we developed and tested an algorithm for the operation of the automatic station CompacT SelecT for the production of large cell mass under standard conditions in order to obtain SFCM from cultured human MSCs (FetMSC cells) after their differentiation in the osteogenic direction. The studies of the effect of secretome concentrate (SFCM) on in vitro cells demonstrated its positive effect on the capacity of MSCs for osteogenic differentiation. It was shown that this method makes it possible to maintain cellular activity, including growth factors that control the ability of cells to differentiate in the osteogenic direction.

\section{ACKNOWLEDGMENTS}

The authors are grateful to Scientific Director of the Biryuch Center for Cell Technologies (EFKO Group) 
(a)

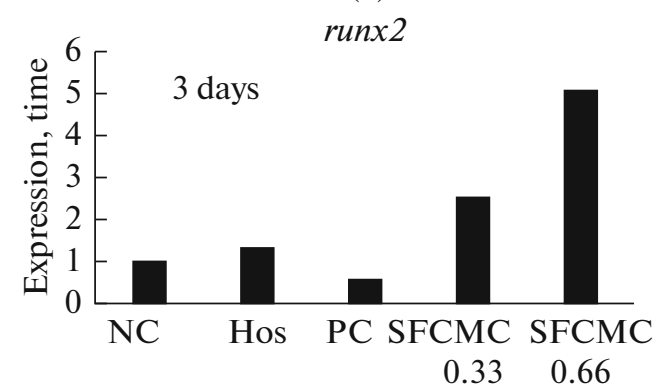

(b)

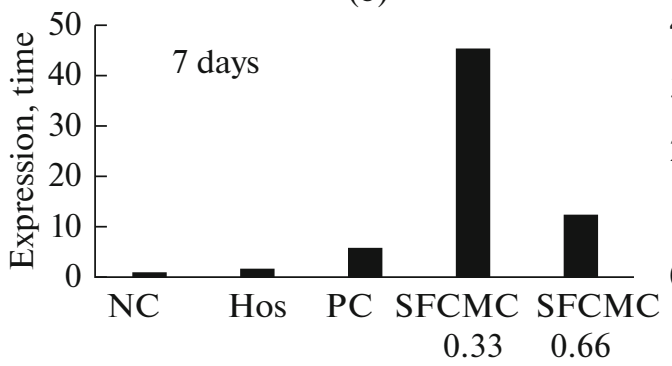

(c)

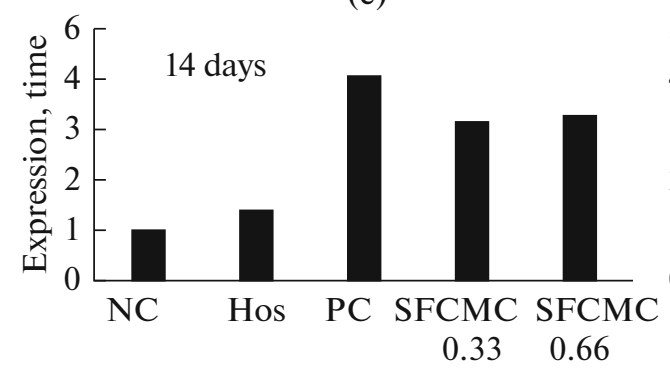

(d)

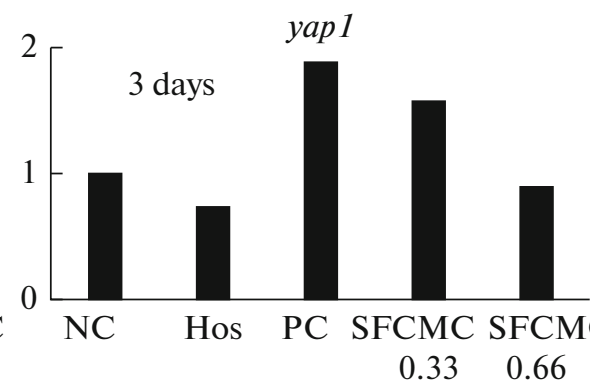

(e)

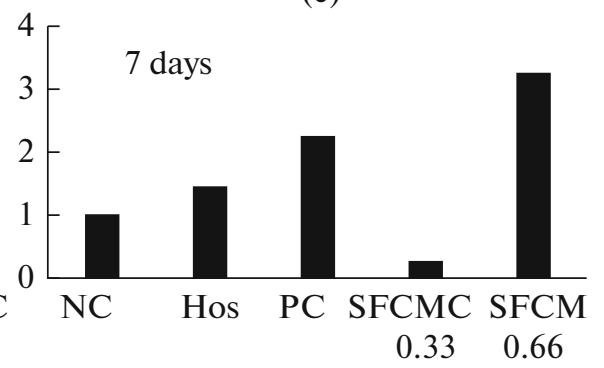

(f)

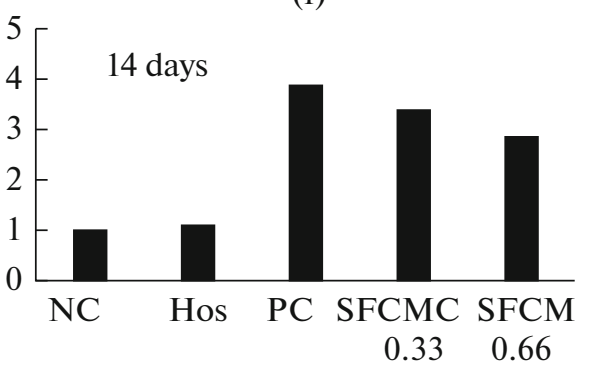

Fig. 5. Expression of $(\mathrm{a}-\mathrm{c})$ runx 2 and $(\mathrm{d}-\mathrm{f})$ yap 1 genes in FetMSC cells in the presence of SFCMC in growth medium (0.33 or $0.66 \mathrm{mg} / \mathrm{mL}$ ) for 3,7 , and 14 days. The differences in gene expression relative to the reference gene ppia is shown. NC, cells cultured under standard conditions (negative control); PC, in a differentiation environment (positive control). Hos, tumor cells.

Yu.E. Burda for the equipment provided for the production of SFCM concentrate.

\section{FUNDING}

This work was supported by the Ministry of Education and Science of Russia (agreement no. 14.575.21.0164, identifier RFMEFI57517X0164, work on the preparation of SFCMC, detection of transcription factors by immunofluorescence and RT-PCR) and the Russian Foundation for Basic Research (project 19-29-04082, work on cultivation and induction osteogenic differentiation of human bone marrow MSCs using the automatic station CompacT SelecT, as well as MSCs live imaging and RNA isolation).

\section{COMPLIANCE WITH ETHICAL STANDARDS}

The authors declare that they have no conflict of interest. This article does not contain any studies involving animals or human participants performed by any of the authors.

\section{REFERENCES}

Assoni, A., Coatti, G., Valadares, M.C., Beccari, M., Gomes, J., Pelatti, M., and Zatz, M., Different donors mesenchymal stromal cells secretomes reveal heterogeneous profile of relevance for therapeutic use, Stem Cells Dev., 2017, vol. 26, p. 206.

Baglio, S.R., Pegtel, D.M., and Baldini, N., Mesenchymal stem cell secreted vesicles provide novel opportunities in (stem) cell-free therapy, Front. Phys., 2012, vol. 3, p. 59.

Caplan, A.I., Mesenchymal stem cells in regenerative medicine, in Principles of Regenerative Medicine, Academic Press, 2019, p. 219.

Da Silva Meirelles, L., Fontes, A.M., Covas, D.T., and Caplan, A.I., Mechanisms involved in the therapeutic properties of mesenchymal stem cells, Cyt. Growth Factor Rev., 2009, vol. 20, p. 419.

Deev, R.V., Drobyshev, A.Y., Bozo, I.Y., and Isaev, A.A., Ordinary and activated bone grafts: applied classification and the main features, Biomed. Res. Int., 2015, p. 365050. https://doi.org/10.1155/2015/365050

Ivanova, G., Pereira, T., Caseiro, A.R., Georgieva, P., and Maurício, A.C., Metabolomic and proteomic analysis of the mesenchymal stem cells' secretome, in Metabolomics- 
Fundamentals and Applications, InTechIntechOpen, eBook. https://doi.org/10.5772/66101

Kim, J.M., Kim, J., Kim, Y.H., Kim, K.T., Ryu, S.H., Lee T.G., and Suh, P.G., Comparative secretome analysis of human bone marrow-derived mesenchymal stem cells during osteogenesis, J. Cell. Phys., 2013, vol. 228, p. 216.

Komori, T., Signaling networks in RUNX2-dependent bone development, J. Cell Biochem., 2011, vol. 112, p. 750.

Krylova, T.A., Koltsova, A.M., Zenin, V.V., Musorina, A.S., Yakovleva, T.K., and Poljanskaya, G.G., Comparative characteristics of new lines of mesenchymal stem cells derived from human embryonic stem cells, bone marrow, and foreskin, Cell Tissue Biol., 2012, vol. 6, p. 95.

Kulterer, B., Friedl, G., Jandrositz, A., Sanchez-Cabo, F., Prokesch, A., Paar, C., Scheideler, M., Windhager, R., Preisegger, K.H., and Trajanoski, Z., Gene expression profiling of human mesenchymal stem cells derived from bone marrow during expansion and osteoblast differentiation, BMC Genomics, 2007, vol. 8, p. 70.

Kusuma, G.D., Carthew, J., Lim, R., and Frith, J.E., Effect of the microenvironment on mesenchymal stem cell paracrine signaling: opportunities to engineer the therapeutic effect, Stem Cells Dev., 2017, vol. 26, p. 617.

Mizukami, A. and Swiech, K., Mesenchymal stromal cells: From discovery to manufacturing and commercialization, Stem Cells Int., 2018, article ID 4083921.

https://doi.org/10.1155/2018/4083921
Murphy, M.B., Moncivais, K., and Caplan, A.I., Mesenchymal stem cells: environmentally responsive therapeutics for regenerative medicine, Exp. Mol. Med., 2013, vol. 45, p. e54. https://doi.org/10.1038/emm.2013.94

Pittenger, M.F., Mbalaviele, G., Black, M., Mosca, J.D., and Marshak, D.R., Mesenchymal stem cells, in Human Cell Culture, The Netherlands: Kluwer Acad. Publishers, 2001, vol. 5, p. 189.

Porter, J.R., Ruckh, T.T., and Popat, K.C., Bone tissue engineering: a review in bone biomimetics and drug delivery strategies, Biotechnol. Prog., 2009, vol. 25, p. 1539.

Stanovici, J., Le, Nail, L.-R., Brennana, M.A., Vidalab, L., Tricheta, V., Rosset, P., and Layrolle, P., Bone regeneration strategies with bone marrow stromal cells in orthopaedic surgery, Curr. Res. Transl. Med., 2016, vol. 64, p. 83.

Sundelacruz, S. and Kaplan, D.L., Stem cell-and scaffold based tissue engineering approaches to osteochondral regenerative medicine, Semin. Cell Dev. Biol., 2009, vol. 20, p. 646.

Yagi, R., Chen, L.F., Shigesada, K., Murakami, Y., and Ito, Y., A WW domain-containing yes-associated protein (YAP) is a novel transcriptional co-activator, EMBO J., 1999, vol. 18, p. 2551.

Zhong, S., He, X., Li, Y., and Lou, X., Conditioned medium enhances osteogenic differentiation of induced pluripotent stem cell-derived mesenchymal stem cells, Tiss. Eng. Regen. Med., 2019, vol. 16, p. 141. 